\title{
Sentiment Analysis of Online Product Reviews Using DLMNN and Future Prediction of Online Product Using IANFIS
}

\section{CURRENT STATUS: UNDER REVISION}

Journal of Big Data $\underline{\underline{Q} \text { Springer }}$

\section{Sasikala $\mathrm{p}$}

Government Science College

shakthesasi@gmail.comCorresponding Author

ORCiD: https://orcid.org/0000-0002-0222-0459

Mary Immaculate Sheela

Pentecost University College

\section{DOI:}

10.21203/rs.2.19872/v1

\section{SUBJECT AREAS}

Medical Informatics

\section{KEYWORDS}

sentiment analysis, sentiment analysis of online product reviews, review analysis, Deep Learning Modified Neural Network (DLMNN), Adaptive Neuro-Fuzzy Inference System (ANFIS), Improved ANFIS (IANFIS) 


\section{Abstract}

Sentiment analysis or opinion mining is one of the major tasks of NLP (Natural Language Processing). It captures the user's opinion, feelings, and belief regarding the respective product especially to determine whether the user's attitude is positive, negative, or neutral. This analysis greatly helps the companies to make necessary changes in their product which in return can overcome the flaws that the product is facing and targets better customer satisfaction. Existing techniques for the sentiment analysis of online product reviews obtained low accuracy and also took more time for training. To overcome such issues in this paper, a DLMNN is proposed for sentiment analysis of online product review and IANFIS is proposed for future prediction of online product. Here, the sentiment analysis and future predictions are done on the products taken from the food review dataset. First, from the dataset, the data values are partitioned into GB, CB, and CLB scenarios and then the review analysis for each scenario is performed separately using DLMNN and they give the result as positive, negative, and neutral reviews for the product. After the process of review classification based on these three scenarios, the future prediction of the products is done by performing weighting factor and classification using IANFIS. Experimental results are compared with some existing techniques and the results show that the proposed method outperforms other existing algorithms.

\section{Full Text}

Due to technical limitations, full-text HTML conversion of this manuscript could not be completed. However, the manuscript can be downloaded and accessed as a PDF. Figures 


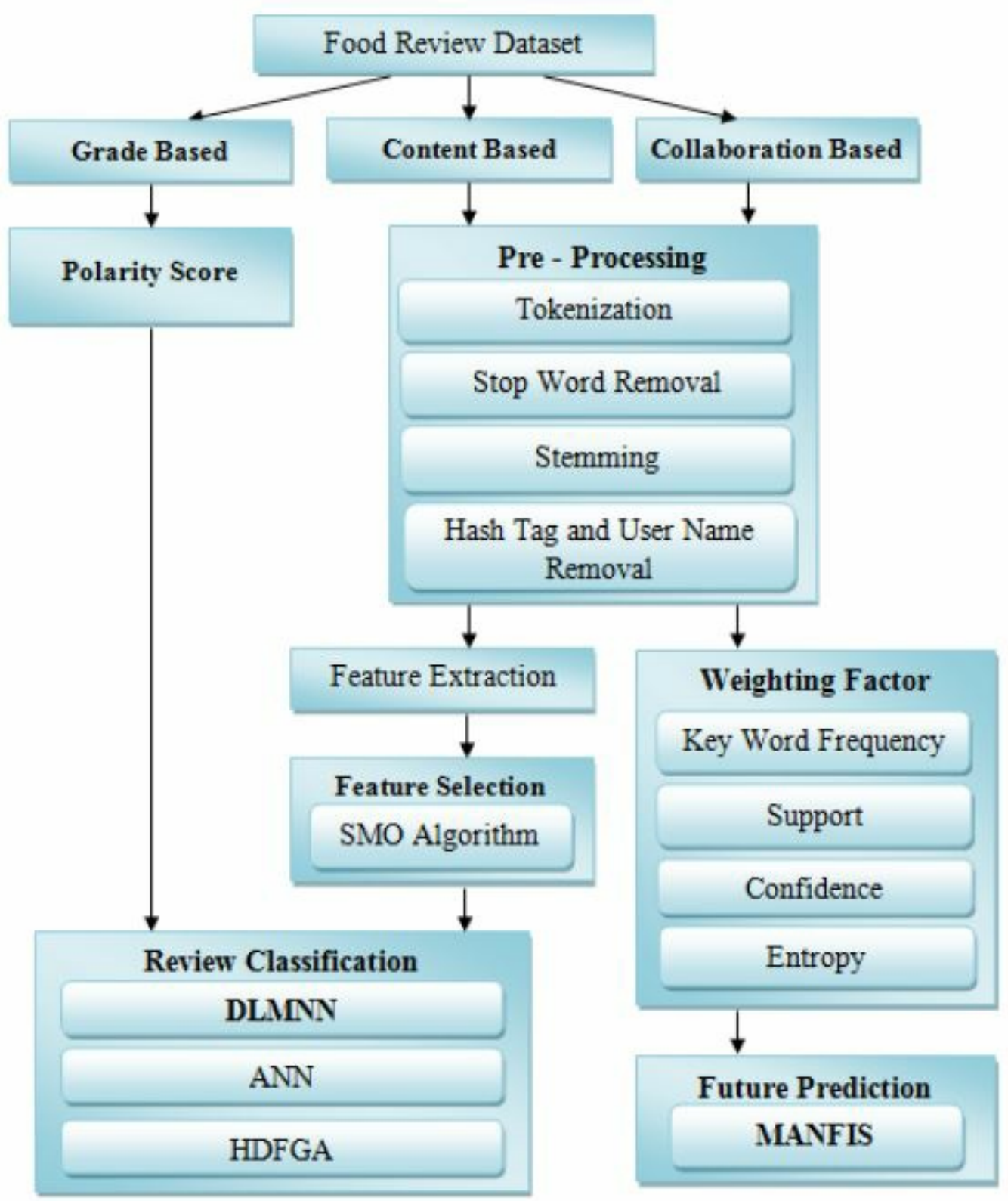

Figure 1

Architecture of the proposed method 
Input: Weight values of DLMNN

Output: Optimized Weight values

\section{Begin}

Initialize the population of dragonflies as $M_{i}=\left(m_{i}^{1}, m_{i}^{d}, \ldots . m_{i}^{N}\right)$

Initialize the step vectors

While the stopping criteria is not met

Calculate the objective function for all $M_{i}$

Calculate $S_{p}, A_{l}, C_{h}, A$, and $D_{r}$

Update the neighborhood radius

If the current dragonfly has at least one neighbouring dragonfly

Select two crossover points

Perform mutation

Else

End if

Update the step vector using equ (24)

Update the position vector using equ (25)

Check and correct the new positions on new boundaries of variables

End while

\section{End}

Figure 2

Pseudocode for the proposed HDF-GA 

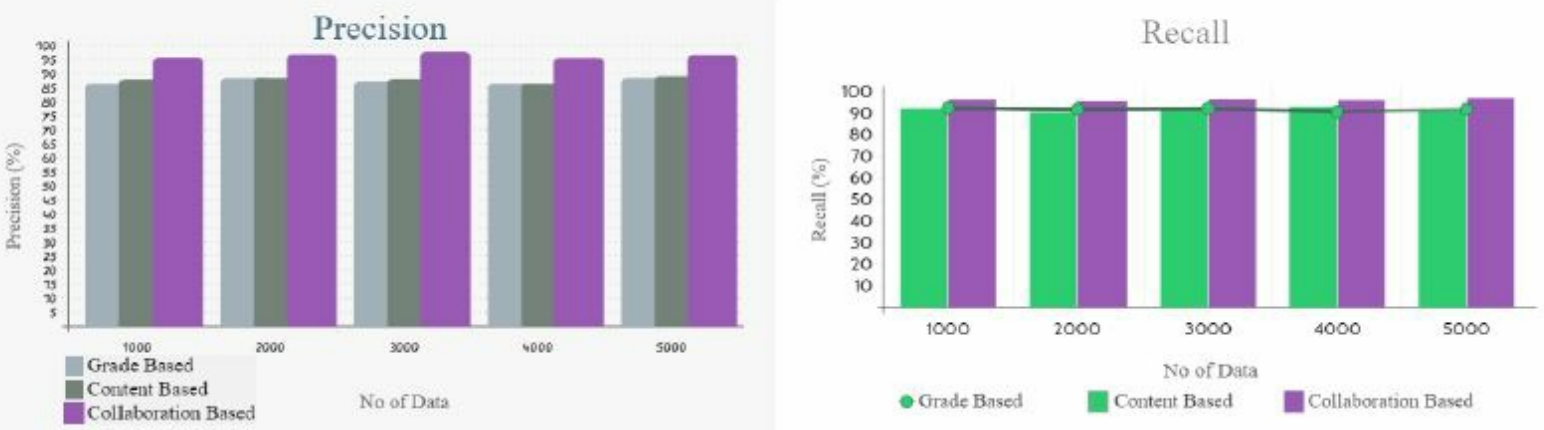

(a)
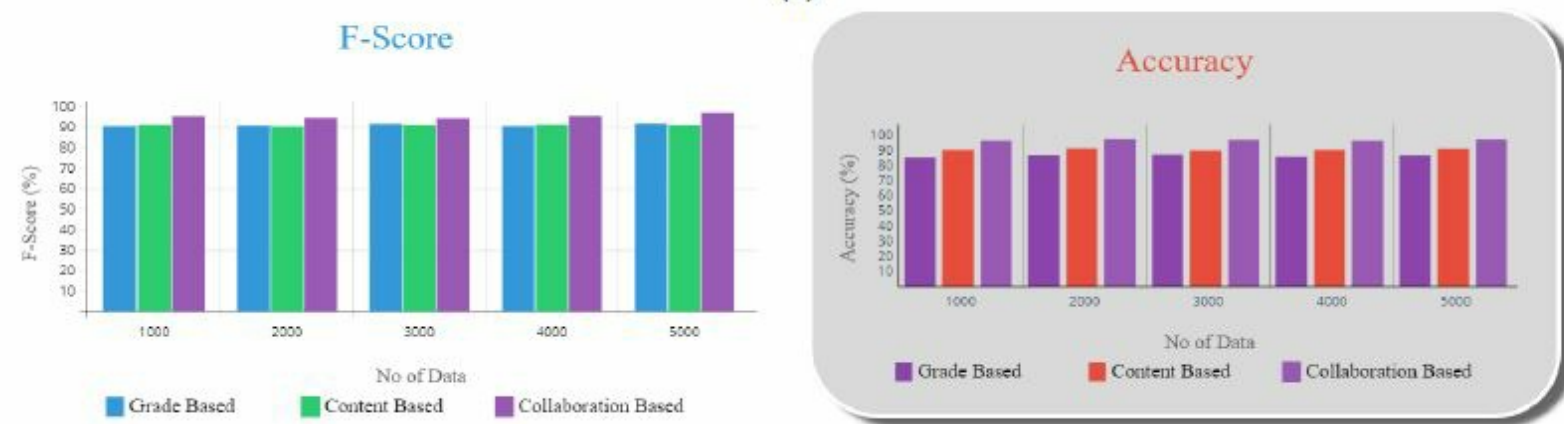

(b)

Figure 3

Performance graph for the GB, CB, and CLB scenario using DLMNN regarding (a) precision and recall, and (b) f-score and accuracy 


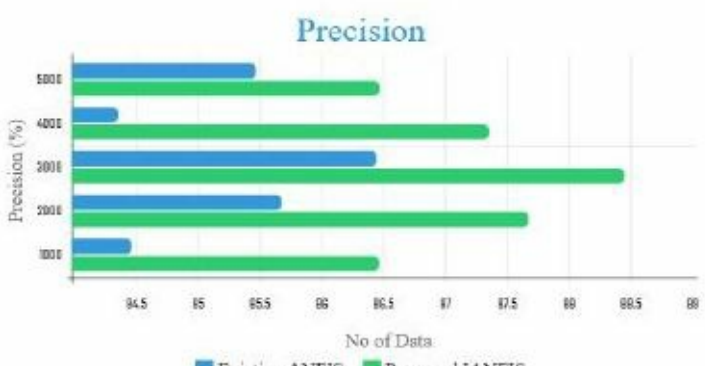

Existing ANFIS Ereposed IANFIS

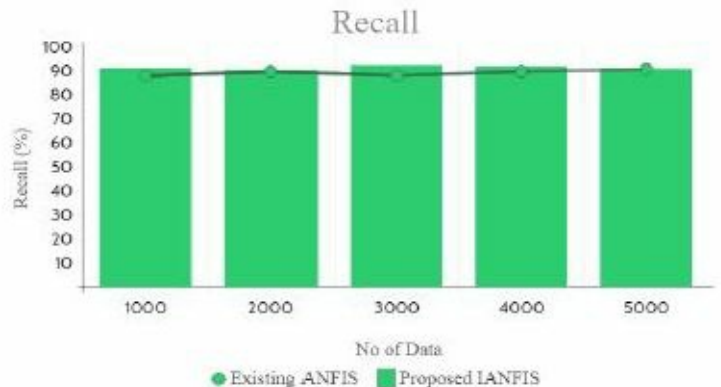

(a)

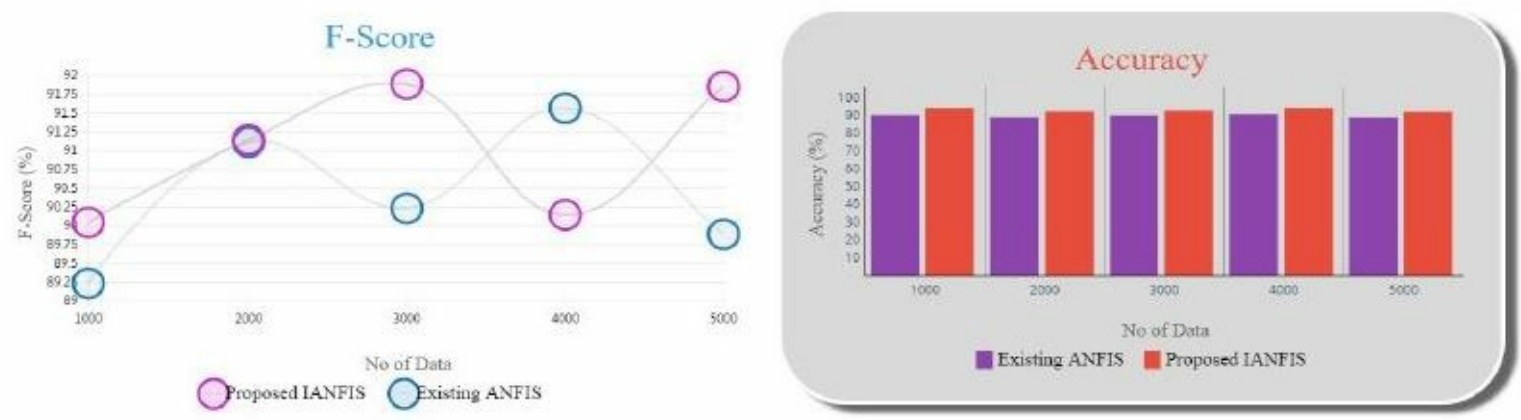

(b)

Figure 4

Performance graph for the IANFIS with existing ANFIS 